\title{
2009 Scientific Referees
}

The quality of the Journal of Orthopaedics and Traumatology depends on the qualified and regular collaboration of renowned scientists who devoted their time to constructively

Paolo Arrigoni (Milan, Italy)

Alexander Berth (Magdeburg, Germany)

Celeste Bertone (Brescia, Italy)

Amin Sadegh Bigham (Shahrekord, Iran)

Andrea Borgo (Vicenza, Italy)

Sergio Brambilla (Milan, Italy)

Massimo Cappella (Modena, Italy)

Shabir A. Dhar (Srinagar, India)

Umberto de Bellis (Milan, Italy)

Cesare Faldini (Bologna, Italy)

Olimpio Galasso (Catanzaro, Italy)

Vincenzo Guzzanti (Rome, Italy)

Tetsuo Hagino (Kofu Yamanashi, Japan)

Takahiko Kiyama (Fukuoka, Japan)

Yoichi Koike (Miyagi, Japan)

Arun Kumar (Cheshire, UK)

Harish V. Kurup (Croydon, UK)

Giulio Maccauro (Rome, Italy)

Bruno Magnan (Verona, Italy)

Cyril Mauffrey (Birmingham, UK) review the submitted articles. We are indebted to the following experts who reviewed papers which completed the peer-reviewing process within 2009.

George Mouzopoulos (Athens, Greece)

Masahiko Nozawa (Tokyo, Japan)

Matthew E. Oetgen (New Haven, CT, USA)

Elisha Ofiram (Rehovot, Israel)

Roberto Padua (Rome, Italy)

Vinay Parmar (Manchester, UK)

Luca Pierannunzii (Milan, Italy)

Filippo Randelli (Milan, Italy)

Faizal Rayan (Kettering, UK)

Enrico Rebuzzi (Oderzo, Italy)

Dario Regis (Verona, Italy)

Fabrizio Rivera (Turin, Italy)

Carlo L. Romanò (Milan, Italy)

Kabul Ch. Saikia (Assam, India)

Celeste Scotti (Milan, Italy)

Roop Singh (Rohtak, India)

Matthias Therbo (Copenhagen, Denmark)

Istemi Yucel (Duzce, Turkey)

Carmine Zoccali (Rome, Italy) 